
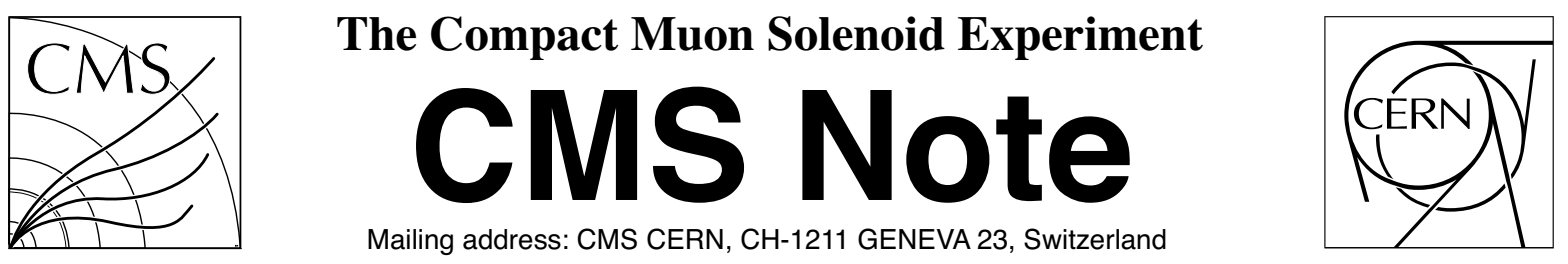

23 February 2011 (v4, 30 June 2011)

\title{
CMS structural equilibrium at constant magnetic field as observed by the Link Alignment System
}

P. Arce, J.M. Barcala, E. Calvo, A. Ferrando, M.I. Josa, A. Molinero, J. Navarrete and J.C. Oller. CIEMAT (Madrid, Spain)

J. Brochero, A.Calderon, M.G. Fernandez, G. Gomez, F.J. Gonzalez-Sanchez, C. Martinez-Rivero, F. Matorras, T. Rodrigo, L. Scodellaro, M. Sobron, I. Vila and A.L. Virto Instituto de Fisica de Cantabria. CSIC-University of Cantabria (Santander, Spain)

J.Fernandez University of Oviedo (Oviedo, Spain)

\begin{abstract}
A study of the time required for the CMS detector to reach its structural equilibrium once the magnetic field is ramped to its operational value of $3.8 \mathrm{~T}$ is presented. In addition, the results from a stability monitoring at $3.8 \mathrm{~T}$ over a nine-month period are given.
\end{abstract}




\section{Introduction}

A major part of the Compact Muon Solenoid detector (CMS) [1-3] is a powerful muon spectrometer for the identification and measurement of muons in a very wide energy range, from a few $\mathrm{GeV}$ up to several TeV. CMS has a cylindrical symmetry around the LHC beam pipe, an overall diameter of $15 \mathrm{~m}$, a total length of $21.6 \mathrm{~m}$ and a total weight of $12.5 \mathrm{kt}$ (mainly iron). At its heart, a $13 \mathrm{~m}$ long, $6 \mathrm{~m}$ inner diameter, $3.8 \mathrm{~T}$ superconducting solenoid, provides a bending power of about $12 \mathrm{Tm}$. The return field is captured through $1.5 \mathrm{~m}$ of iron, allowing 4 muon stations to be integrated to ensure robustness and full geometrical coverage.

Depending on the magnet field intensity and homogeneity, two different technologies are employed. In the barrel region, surrounding the coil of the solenoid, four layers of drift tube (DT) chambers (named MB1 to MB4), interleaved with the return iron yoke, make a redundant momentum measurement. A muon chamber is made of three superlayers. Each superlayer is, in turn, made of four layers of drift cells. The drift cell is the basic unit, measuring drift time and providing a spatial resolution of $250 \mu \mathrm{m}$. Superlayers are arranged such that they measure the spatial position of muon track "segments" along one coordinate: two superlayers measure the $\mathrm{R} \Phi$ coordinate and one superlayer measures the $\mathrm{Z}$ coordinate. The mechanical design of a drift chamber is driven by the spatial precision requirement in the determination of a segment, $100 \mu \mathrm{m}$, which is obtained by a fit to the individual hits in each cell. The muon drift chambers are subject to variable residual magnetic fields, below $0.4 \mathrm{~T}$ for all the chambers except for the innermost MB1 chambers $(0.8 \mathrm{~T})$ near the endcaps. It is worth to notice that the DT chambers provide also the L1 trigger and the bunch crossing identification.

In addition to the DT chambers there are also six layers of Resistive Plate Chambers (RPCs): two around stations 1 and 2 and one in the inner side of stations 3 and 4 that contributes to the collection of the muon track hits.

At both CMS endcap sides there are four layers of muon chambers, named ME1 to ME4. In the endcap regions the magnetic field is typically high but also very inhomogeneous due to its bending to feed the barrel yoke. To cope with that and with the high particle fluxes CMS uses in these regions other gas ionization detectors called Cathode Strip Chambers (CSCs) which are multi-wire proportional chambers in which one cathode plane is segmented into strips running across wires, giving $2 \mathrm{D}$ information of the particle passage. Due to the intense magnetic field, the muon trajectories bend more in the vicinity of the first endcap station, where a higher precision is required (75 $\mu \mathrm{m})$. For the rest of the chambers the needed precision is about $150 \mu \mathrm{m}$.

The measurement of the muon momentum is related to its bending in the transverse plane. The radius of curvature $\rho$ and the momentum of a muon perpendicular to the magnetic field $\left(\mathrm{p}_{\mathrm{t}}\right)$ are related by $\rho[\mathrm{m}]=\mathrm{p}_{\mathrm{t}}[\mathrm{GeV} / \mathrm{c}] / 0.3 \mathrm{~B}[\mathrm{~T}]$. The radius of curvature can be obtained from the measurement of the muon trajectory sagitta $s$, after traversing a distance $d$ in the magnetic field, using the approximate expression $\rho=d^{2} / 8$ s. An error in the sagitta measurement results in an error in the momentum measurement. The relative error in the sagitta measurement is $\delta \mathrm{s} / \mathrm{s}=\delta \mathrm{p}_{\mathrm{t}} / \mathrm{p}_{\mathrm{t}}$, proportional to $\sigma_{\mathrm{s}}[\mathrm{mm}] \mathrm{p}_{\mathrm{t}}[\mathrm{TeV}] / \mathrm{d}^{2}\left[\mathrm{~m}^{2}\right] \mathrm{B}[\mathrm{T}]$, where $\sigma_{\mathrm{s}}$ is the resolution in the sagitta measurement. The relative resolution deteriorates with the muon momentum and improves linearly with the magnetic field and quadratically with the traversed distance. 
The accuracy required in the position of the muon chambers is determined by the resolution demanded in the momentum reconstruction of high energy muons. CMS is designed to achieve a combined (Tracker [4] and Muon System) momentum resolution for the region $|\eta|<2.4$ of $0.5-1 \%$ for $\mathrm{p}_{\mathrm{t}} \approx 10 \mathrm{GeV}, 1.5-5 \%$ for $\mathrm{p}_{\mathrm{t}} \approx 100 \mathrm{GeV}$ and $5-$ $20 \%$ for $\mathrm{p}_{\mathrm{t}} \approx 1 \mathrm{TeV}$. This design accuracy requires the knowledge of the position of the chambers with a precision comparable to their resolution.

Several simulation studies have been performed [5] in order to quantify the importance of muon chamber alignment in the momentum resolution. For the most important coordinate from the physics point of view, $(R \Phi)$, the alignment system should reconstruct the position of the chambers within the $150-300 \mu \mathrm{m}$ range for MB1 - MB4 and within the $75-200 \mu \mathrm{m}$ range for ME1 - ME4. The tighter constraints correspond to ME1 and MB1 since most of the muons change the sign of its curvature near the first muon stations. Since these stations are located at the border of the magnet they allow, in combination with the Tracker hits, to exploit the full bending space in the CMS experiment.

However, when CMS is in operation, the movements and deflections of the muon spectrometer exceed $100 \mu \mathrm{m}$. To monitor these movements, CMS is instrumented with an opto-mechanical alignment system that allows a continuous and precise measurement of the relative position of the muon chambers amongst themselves as well as the position of the muon chambers with respect to the tracker, assumed to be a rigid body. The information provided by the alignment is used for off-line corrections in the track reconstruction.

During the years 2008 and 2009, prior to the restart of LHC, CMS recorded cosmic ray data at $3.8 \mathrm{~T}$, chosen as the working magnetic field intensity, in many runs called CRAFT08 and CRAFT09. The name CRAFT stands for Cosmics Run at Four Tesla [68]. The Link Alignment System [3] was used to monitor displacements, rotations and deformations of relevant mechanical structures. A previous document [9] analyzed the motions observed by the Link Alignment System caused by the ramp up in magnetic field intensity from $\mathrm{B}=0 \mathrm{~T}$ to $\mathrm{B}=3.8 \mathrm{~T}$. The present work studies the stabilization of mechanical structures from the moment in which the magnetic field reaches its nominal working strength, in order to estimate the time the CMS detector takes to reach mechanical equilibrium.

In addition, some of the Link System sensors were read out over long time periods during the physics data taking runs in 2010, allowing to check the assumed mechanical stability when the magnetic field is at a constant strength of $\mathrm{B}=3.8 \mathrm{~T}$.

This document is organized as follows: an introduction to the CMS Alignment System is given in section 2. The data used to determine the stabilization time once the $3.8 \mathrm{~T}$ magnetic field is reached are presented in Section 3, while the analysis and results are given in Section 4. A survey of changes in the mechanical conditions along sequenced Link System runs over a nine-month period in 2010 is shown and discussed in Section 5. Finally, summary and conclusions are presented in Section 6.

\section{The CMS Alignment System}

At 3.8 $\mathrm{T}$ the solenoid induces an axial force of about 10,000 ton on the endcap iron yokes in the direction of the centre of the solenoid. Aluminium blocks, called Z-stops, prevent the endcap disks from getting pushed into each other and crash onto the barrel 
wheels. A longitudinal view of one quadrant of the CMS experiment is shown in Fig. 1, where the various detectors and the position of the Z-stops are indicated.

The muon system detection planes embedded in the $15 \mathrm{~m}$ diameter, $20 \mathrm{~m}$ long return yoke allow to get, typically, about 50 points per muon track, including the PIXELs ones. In order to meet the muon momentum resolution requirements mentioned above, the system is instrumented with an Alignment System organised in three basic blocks:

- Tracker alignment system, to measure the position of the various tracker modules and monitor eventual internal deformations.

- Muon System (Barrel and Endcaps) alignment, to monitor the relative positions among the DT and CSC muon chambers.

- Link System, to relate the position of the various elements of the muon system (Barrel and Endcaps) to the position of the tracker body, and to monitor the relative movements between both systems.

Several types of alignment sensors are supported by a series of independent reference rigid bodies which are individually calibrated and intercalibrated on special benches and measured by photogrammetry when in place in CMS. The position of the sensors is such that when the structures are in place they define three planes. The left side of Fig. 2 shows one of the $\Phi$ alignment planes where the three alignment subsystems can be seen. Each plane contains four independent alignment quadrants where the three systems are connected. The three $\Phi$ planes are also depicted on the right side of Fig. 2, where the CMS coordinates-system is also indicated. Sketch of a quadrant of $\Phi$ alignment plane with its instrumentation is shown in Fig. 3.

Details about the CMS Alignment System can be found in [3, 4, 9 and 10]. The Link Alignment System is briefly described below.

The distributed network of Amorphous Silicon Position Sensors (ASPDs) in each quadrat is connected by laser lines. An ASPD sensor [11-13] consists of two groups of 64 silicon micro-strips $408 \mu \mathrm{m}$ wide, with a pitch of $430 \mu \mathrm{m}$, oriented perpendicularly. The intrinsic sensor position resolution is better than $5 \mu \mathrm{m}$.

The entire Link System is divided into three $\Phi$ planes $60^{\circ}$ apart starting at $\Phi=15^{\circ}$. As said, each plane consists of four independent quadrants, resulting in 12 laser paths, or lines: 6 on each side (positive or negative Z) of the CMS detector. Three laser light paths originate at Tracker, Endcap, and Barrel regions as indicated in Fig. 3. All lasersource collimators are housed in rigid carbon fibre structures: Alignment Rings (ARs), Modules for the Alignment of the Barrel (MABs), and Link Disks (LDs).

The ARs are annular structures placed at both ends of the tracker. The LDs are suspended from the outer diameter of the YN1 iron disks of the end-cap muon spectrometer by means of aluminium tubes attached to mechanical assemblies called Transfer Plates (TPs). MABs are mounted onto the barrel yokes.

The laser-ASPD measurement network is complemented by electrolytic tiltmeters (for angular measurements with respect to the gravity vector), optical and mechanical proximity sensors for short distance measurements, aluminium bars (longitudinal and radial profiles, labelled LP and RP) for long distance measurements, magnetic probes and temperature sensors, also shown in Fig. 3. Changes in length of the aluminium profiles due to temperature variations are controlled by the readout of the appropriate temperature probes.

Three $3600 \mathrm{~mm}$ long LP relate $\mathrm{LD}$ and AR. On the end closest to the AR, a target is in contact with a potentiometer [14] located at the AR, allowing to monitor the relative 
distance between LD and AR structures along the CMS Z coordinate at three different $\Phi$ positions. The relative $\mathrm{Z}$ distance between the TP and the ME/1/1 chamber is also measured by a contact potentiometer installed in the TP touching a target situated on the top side of the ME/1/1 chamber (see Fig 3).

The rest of the relative distance measurements between CMS elements in a $\Phi$ quarter monitor motions in the $\mathrm{R}$ direction. The longest monitored radial distance, between LD and TPs, is measured using a $1977 \mathrm{~mm}$ long radial profile (RP in Fig. 3) instrumented with a potentiometer in the end closest to the LD. The relative displacement between the $\mathrm{TP}$ and the bottom side of the ME/1/2 chamber is also monitored using a contact potentiometer. The relative $\mathrm{R}$ distance between the $\mathrm{MAB}$ and the outer side of the $\mathrm{ME} / 1 / 2$ chamber in the corresponding $\Phi$ quadrant is monitored using a non-contact proximity sensor (Omron [15]) installed at the innermost part of each MAB structure. The sensor emitting/receiving head directs a laser light and receives the reflected light to/from a reflective target located on the outer region of the ME/1/2 chamber. All proximity measurements along $\mathrm{R}$ are labelled as Distancemeter $\mathrm{R}$ in Fig. 3.

The estimated combined uncertainty (mechanical support length plus proximity sensor output plus the mounting uncertainty) in the measurement of absolute positions is about $305 \mu \mathrm{m}$. Nevertheless, relative distance measurements, which are the relevant ones for this note, are only affected by the precision of the proximity sensors, $\sim 40 \mu \mathrm{m}$.

As already said and complementing the laser and distance measurement system, all the alignment structures (ARs, LDs, TPs, and MABs) are instrumented with different models of tiltmeter sensors [16] which provide direct information on changes in orientations (small rotations/tilts). The precision of these sensors is of the order of $30-$ $40 \mu \mathrm{rad}[17]$. With these devices, the Link Alignment System can monitor changes in the CMS $\Phi$ (azimuthal angle, rotations around the Z-axis) and $\Theta$ (polar angle, rotations around the X-axis) angles of the AR, BD (Back Disk, the outermost, uninstrumented Tracker Endcap disc), LD and TP structures. In the case of the ARs and the BDs, for instance, it is possible to detect rotations $(\Phi)$ and/or bends $(\Theta)$ of the Tracker body. In the case of the LDs, they detect rotations and/or bends of the YN1 endcap iron disks.

For the MAB structures the only monitored angle is $\Phi$. Fig. 4 shows a sketch of a MAB with the position of the tiltmeter attached to it. The sensor is placed in an $\mathrm{X}-\mathrm{Y}$ plane in order to detect a rotation of the structure around the $+Z$ axis. Rotations would be registered as small variations ( $\mu \mathrm{rads}$ ) around the nominal $\Phi$ value of each particular $\mathrm{MAB}$ and would correspond to eventual tilts of the muon barrel wheels.

\section{CMS structural stabilization time at the working magnetic field}

Link data recorded in one run from CRAFT08 and one run from CRAFT09 are used to study the stabilization time (Ts) at the working field of $\mathrm{B}=3.8 \mathrm{~T}$. Ts is defined as the time after which any further motion in a given run is smaller than or equal to the sensors resolutions in measuring displacements $(40 \mu \mathrm{m})$ and tilts $(30 \mu \mathrm{rad})$. Both runs consisted of a ramp from $\mathrm{B}=0 \mathrm{~T}$ to $3.8 \mathrm{~T}$ followed by several hours at stable $3.8 \mathrm{~T}$ field. The CRAFT08 run started on 27/10/08 at 09:25 h, lasting about $29.3 \mathrm{~h}$, with 355 events recorded, 299 of them taken at $\mathrm{B}=3.8 \mathrm{~T}$. A Link System event is defined as one complete set of recorded data from the Link System readout. Data were taken at a rate of one event every five minutes. The chosen CRAFT09 run started one year later, on 
27/10/09 at 7:45 h, lasting about $29.5 \mathrm{~h}$ with 354 events recorded, out of which 256 were taken at $\mathrm{B}=3.8 \mathrm{~T}$. The data taking rate was the same as in CRAFT08. Figs. 5 a) and $b$ ) show the magnetic field intensity as a function of the event number in both CRAFT08 and CRAFT09 runs, respectively.

During these two runs and in order to speed-up the data taking, no information from the ASPDs was recorded: a full turn-around of photo-sensors information takes about $27 \mathrm{~min}$. From the rest of the many sensors composing the Link Alignment System, two sets monitoring axial displacements, two sets monitoring radial displacements, and one set monitoring rotations in the $\Phi$ angle were chosen for Ts calculation. The analyzed parameters were:

- the relative axial distance $(\Delta \mathrm{Z})$ between the LD and the AR at the six equipped angles, $\Phi=+/-75^{\circ}, 195^{\circ}$ and $315^{\circ}$ (the sign refers to the CMS Z side) and between the TP and the ME/1/1 muon chamber at the twelve $\Phi$ quadrants (six per $\mathrm{Z}$ side)

- the relative radial distance $(\Delta \mathrm{R})$ between the MAB structure and the ME/1/2 muon chamber and between the TP and the same ME/1/2 chamber (in both cases at the twelve $\Phi$ quadrants)

- the relative tilt angle $\Delta \Phi$ at the twelve external MABs (six per CMS Z side)

\subsection{The general trend of motions during the ramping up of the magnetic field}

The mechanical motions during the ramping up of the CMS magnetic field from $0 \mathrm{~T}$ to $3.8 \mathrm{~T}$ are roughly similar [9], but far from identical, for the analyzed runs. The LD approaches its corresponding AR due to the motion of the YN1 yoke (to which the LD is attached) by $13-14 \mathrm{~mm}$ in the $\mathrm{Z}$ direction, caused by the magnetic force pushing the end-cap yokes towards the CMS geometrical center at both $\mathrm{Z}$ sides. Figures $5 \mathrm{c}$ ) and d) illustrate that relative motion at $\Phi=+75^{\circ}$, as a function of the event number (labeled as data number in the figure), in CRAFT08 and CRAFT09 runs, respectively. The ME/1/1 muon chambers move $1.5-2.5 \mathrm{~mm}$ in the $\mathrm{Z}$ direction, away from their corresponding TP structure. The Z-stops prevent the crash between the endcaps and the barrel (approximate positions of the Z-stops in a CMS sextant are depicted in Fig. 1). The motion is illustrated in Figs. $5 \mathrm{e}$ ) and f) for the $\Phi=-315^{\circ}$ quadrant in both runs.

The ME1/2 muon chambers move radially outwards, approaching their corresponding MAB structure by $2-4 \mathrm{~mm}$ as shown in Figs. 6 a) and b) for the $\Phi=+15^{\circ}$ quadrant, and separating from their corresponding TP structure by about $3 \mathrm{~mm}$ as shown in Figs. $6 \mathrm{c}$ ) and d) for the $\Phi=-15^{\circ}$ quadrant, in both CRAFT runs.

The MAB tilt sensors indicate a very small clockwise rotation $(\Delta \Phi$ is always smaller than $100 \mu \mathrm{rad}$ ), around the CMS Z coordinate, as illustrated in Figs. $6 \mathrm{e}$ ) and $\mathrm{f}$ ) for the MAB at $\Phi=+15^{\circ}$, in both runs.

\section{Calculation of Ts: method and results}

In the CRAFT08 run, the magnetic field reached an intensity of $\mathrm{B}=3.8 \mathrm{~T}$ at event number 56, about $4.7 \mathrm{~h}$ after the start of the magnet ramp up. For CRAFT09, the field 
reached $3.8 \mathrm{~T}$ at event 98 , about $8.2 \mathrm{~h}$ after the start of the run. These two events will be taken as the start time, $\mathrm{t}_{\mathrm{o}}=0 \mathrm{~h}$, for the determination of Ts. Figures 7 and 8 show the measured axial (Figs. 7 a) to d)), radial (Figs. 8 a) to d)) and angular (Figs. 8 e) and f)) motions for the indicated $\Phi$ quadrants in both CRAFT runs as a function of the elapsed time after $t_{0}$.

For each of the 54 relative motions measured, iterations are made in time intervals $\geq 1$ hour, starting at a given time $t_{0}{ }^{\prime}$. We study the distributions of the quantities:

$$
\Delta\left(\text { variable }_{\mathrm{j}}\right)_{\mathrm{i}}=\left(\text { measured variable } \mathrm{j}_{\mathrm{j}} \text { at } \mathrm{t}=\mathrm{t}_{\mathrm{o}}{ }^{\prime}+\Delta \mathrm{t}\right)_{\mathrm{i}}-\left(\text { measured variable } \mathrm{j}_{\mathrm{j}} \text { at } \mathrm{t}=\mathrm{t}_{\mathrm{o}}{ }^{\prime}\right)_{\mathrm{i}}
$$

where $t_{o}$ ' takes, in sequence, the values $0,1,2, \ldots$ hours, " $\mathrm{j}$ " refers to each of the 54 relative motions used, and " $i$ " extends over all events recorded from $t=t_{0}$ ' onwards (in steps of $\Delta t=5 \mathrm{~min}$, the fixed time interval between two consecutive measurements).

The extreme values $\Delta \min$ and $\Delta \max$ of these distributions are, of course, a function of $\mathrm{t}_{\mathrm{o}}{ }^{\prime}$. As mentioned earlier, Ts is determined as the time $\mathrm{t}_{\mathrm{o}}{ }^{\prime}$ for which $|\Delta \mathrm{min}|$ and $|\Delta \max |$ are smaller than the resolution of the corresponding readout sensor: $40 \mu \mathrm{m}$ in the case of the Sakae potentiometers or Omrons proximity sensors, and $30 \mu \mathrm{rad}$ in the case of the AGI tilt sensors.

For example, let us study the variable $\Delta \mathrm{Z}(\mathrm{LD}-\mathrm{AR})$ at $\Phi=+315^{\circ}$ from the CRAFT09 run. The distributions of the measurements for $\mathrm{t}_{\mathrm{o}}{ }^{\prime}=0,2,3$ and $4 \mathrm{~h}$ are shown in Figs. 9 a) to d), respectively. Table 1 shows the mean, RMS, $\Delta$ min and $\Delta$ max values corresponding to these distributions. For this variable, the structural equilibrium occurs in the interval $t_{0}^{\prime}=2-3 \mathrm{~h}$. Since the study uses one-hour intervals, half an hour is taken as the average error in Ts and therefore Ts $=2.5 \pm 0.5 \mathrm{~h}$ is determined for this observable.

This exercise is repeated for the 54 variables and for both runs under consideration; the results are presented in Table 2. It is important to note that each variable is measured at various $\Phi$ positions. For instance $3+3$ in the case of $\Delta \mathrm{Z}(\mathrm{LD}-\mathrm{AR})$ and $6+6$ in the case of $\Delta \Phi(\mathrm{MAB})$. The values of Ts shown in Table 2 correspond to the $\Phi$ positions taking the longest stabilization time.

Table 2 suggests that, once the magnetic field reaches $\mathrm{B}=3.8 \mathrm{~T}$, the CMS detector will reach its structural equilibrium about $13.5 \pm 0.5$ hours later. This time corresponds to the moment in which event number 218 was recorded in the CRAFT08 run and event number 260 was recorded in the CRAFT09 run used in this analysis. Taking the values recorded for these events as new references, the maximum expected motions in $\Delta \mathrm{Z}, \Delta \mathrm{R}$ and $\Delta \Phi$ after stabilization are calculated, as shown in Table 3. Once CMS reaches mechanical equilibrium, further motions will not exceed $\pm 26 \mu \mathrm{m}$ in the $\mathrm{Z}$ direction and $\pm 31 \mu \mathrm{m}$ in the R direction, while the tilts in $\Phi$ will not exceed $\pm 30 \mu \mathrm{rad}$.

\section{A long-term monitoring of the CMS stability}

CMS is in full operation since March 2010. Since then, the magnet was switched on and off in several occasions. Data recorded by the Link system over the period from March to October of 2010 were analyzed, in order to check the CRAFT08 and CRAFT09 results. 


\subsection{Monitored periods and monitored Link sensors}

The first data taking followed the initial magnet ramp from $0 \mathrm{~T}$ to $3.8 \mathrm{~T}$ after the closing of CMS at the beginning of March 2010 for the $7 \mathrm{TeV}$ pp-collisions physics run. As said, over the nine monitored months several magnet on-and-off cycles occurred.

A period is defined as the elapsed time between a magnet on and a magnet off operation. The different periods analyzed, the starting and ending date and the duration of every period are detailed in Table 4. Only data collected at least $24 \mathrm{~h}$ after the magnetic field reached $3.8 \mathrm{~T}$ is used in the following analysis. Therefore, according to the results obtained from CRAFT08 and CRAFT09 Link System data, CMS would have already reached mechanical stability and motions beyond the sensors resolutions, $40 \mu \mathrm{m}$ for distances and $30 \mu \mathrm{rad}$ for tilts would be not expected.

The following Link related variables were used:

- the changes in the axial distance $(\Delta Z)$ between the LD and the AR at the six equipped angles, $\Phi=+/-75^{\circ}, 195^{\circ}$ and $315^{\circ}$ (the sign refers to the CMS Z side) and between the TP and the ME1/1 muon chamber at the twelve $\Phi$ quadrants (six per $\mathrm{Z}$ side)

- the changes in the relative radial distance $(\Delta \mathrm{R})$ between the TP and the nearest ME1/1 chamber, between TP and the nearest ME1/2 chamber and between the LD and the corresponding TP, all three observables at the twelve $\Phi$ quadrants

- the variations in the relative tilt angles $\Delta \Phi$ and $\Delta \Theta$ of the AR and LD structures at both $\mathrm{Z}$ sides (six parameters per angle in both cases) and the relative tilt angles $\Delta \Phi$ at the twelve external MABs (six per CMS Z side)

\subsection{Results from the observations and discussion}

For each of the 66 relative motions measured, and in every monitored period, the distribution of the quantities:

$$
\Delta\left(\text { variable }_{j}\right)_{i}=\left(\text { measured variable } \text { at time } \mathrm{t}_{i}\right)-\left(\text { measured variable }{ }_{j} \text { at } \mathrm{t}=0\right)
$$

is evaluated; " $j$ " refers to each of the 66 relative motions observed, and " $i$ " extends over all events recorded from $t=0$ onwards, i.e. with respect to the first recorded event in every time period. The mean value $\left\langle\Delta\left(\right.\right.$ variable $\left.\left._{j}\right)\right\rangle$ and the RMS of the distributions are extracted. As mentioned earlier, the expectations were to find, for all periods, distributions such that the absolute value of $\left\langle\Delta\left(\right.\right.$ variable $\left.\left._{j}\right)\right\rangle \pm$ RMS were smaller than the resolution of the corresponding readout sensor.

Data showed that, during the nine-month long monitoring at 3.8 T, the CMS structural stability was observed in all of the analyzed variables except for those related to the relative distance between the LDs and the ARs. The motion of the endcap iron yokes towards the Tracker is the most affected by the magnetic field strength variation when ramping from $0 \mathrm{~T}$ to $3.8 \mathrm{~T}$.

The most unstable period was P1, the one following the CMS closing. From the 66 observables in the seven analyzed time periods, the measurements with largest deviation from zero in period P1 are shown in Table 5, compared to the same measurements for 
periods $\mathrm{P} 2$ to $\mathrm{P} 7$. In period $\mathrm{P} 1, \Delta \mathrm{Z}(\mathrm{LD}-\mathrm{AR})$ at $\left(+\mathrm{Z}, \Phi=315^{\circ}\right)$ exhibits an average motion incompatible with mechanical structural stability. In later periods, P2 to P7, this observable is compatible with mechanical stability at the level of 2 standard deviations. Fig. 10 shows the measurements of this observable, taking as a reference the sensor value in the first event of the period, for P1, P3, P5 and P7, as a function of an internal Link System run number (a.u. in the figure). Each unit has a time-length of 1 day. A maximum of two full events are recorded each day, but there were some Link runs were no data taken took place. In the figure, most of the black dots are the overlap of two events recorded in a given Link run.

Extensive information can be extracted from Fig. 10. First, no repetitive behavior is observed; CMS behaves differently in every period. Periods P2, P4 and P6 are not included in the figure for the sake of clarity, but they are also different from the ones shown. During period P1 (40 days of observation) the endcap moves continuously towards the Tracker without reaching equilibrium. The aluminum of the corresponding Z-stop is sagging under the pressure of the YE1 yoke during the entire period (a total displacement of about $200 \mu \mathrm{m}$ ). In period P3 instead, the Z-stop rejects/stags the pressing of the YE1 iron, with alternating values $\Delta \mathrm{Z} \approx 0 \mu \mathrm{m}$ and $\Delta \mathrm{Z} \sim 100 \mu \mathrm{m}$. Period P5 exemplifies the expected behavior of clear mechanical stability as no motion beyond $40 \mu \mathrm{m}$ is observed. Finally, in period P7 (45 days of observations) the initial stability is broken at day 14 , when YE1 approaches the CMS tracker by $\approx 50 \mu \mathrm{m}$ (sagging of the corresponding Z-stop aluminum structure) before reaching equilibrium.

It is important to note that the observed jumps in Fig. 10, above sensors resolutions, are real motions, not instrumental problems.

The measured tilt variations (with respect to the first recorded data in each period) over the same four periods for the MAB at $\left(-Z, \Phi=15^{\circ}\right)$ are given in Fig. 11, showing a fully stable situation. All observed rotations (probably due to small temperature changes) are compatible with zero within the $30 \mu \mathrm{rad}$ resolution of the tilt-sensor.

\section{Summary and conclusions}

Using Link Alignment data recorded during one CRAFT08 run and one CRAFT09 run (spaced by one year), the relative motions between various CMS mechanical structures were analyzed. The results suggested that the CMS detector stabilizes $13.5 \pm$ 0.5 hours after the magnetic field reaches the nominal intensity of $\mathrm{B}=3.8 \mathrm{~T}$, given that displacements between mechanical structures beyond this time do not exceed the sensors resolutions.

A later study performed in 2010 for seven different time periods over nine months to measure motions at constant $\mathrm{B}=3.8 \mathrm{~T}$ magnetic field showed that the expected mechanical stability is not observed everywhere. In particular, the relative distance $\Delta \mathrm{Z}(\mathrm{LD}-\mathrm{AR})$ in all of the six $\Phi$ sectors exhibits variations larger than the sensor resolutions in most of the analyzed periods thus questioning the idea of an irrevocable structural equilibrium. However, the observed out-of-stability variations do not exceed $\sim 200 \mu \mathrm{m}$ and therefore its impact on the muon $\mathrm{p}_{\mathrm{t}}$ resolution will be negligible. 


\section{References}

[1] CMS Collaboration, "The CMS experiment at the CERN LHC", JINST 3 (2008) S08004.

[2] The CMS Collaboration, "The Magnet Project Technical Design Report", CERN/LHCC 97-10.

[3] The CMS Collaboration, "The Muon Project Technical Design Report", CERN/LHCC 97-32.

[4] The CMS Collaboration, "The Tracker Project Technical Design Report", CERN/LHCC 98-06.

[5] V. Karimaki, and G. Wrochna, CMS TN/94-199; F. Matorras and A. Meneguzzo, CMS TN/95-069 and I. Belotelov et al. CMS NOTE 2006/017.

[6] The CMS Collaboration, "Commissioning of the CMS experiment and the cosmic run at four tesla", 2010 JINST 5 T03001.

[7] The CMS Collaboration, "Aligning the CMS muon chambers with the muon alignment system during an extended cosmic ray run", 2010 JINST 5 T03019.

[8] The CMS Collaboration, "Alignment of the CMS muon system with cosmic-ray and beam-halo muons", 2010 JINST 5 T03020.

[9] L.A. García Moral et al., Nucl. Instr. And Methods A 606 (2009) 344.

[10] M. Hohlmann et al., "Design and Performance of the Alignment System for the CMS Muon Endcaps". CMS CR-2008/016

[11] A. Calderón et al., Nucl. Instr. and Methods A 565 (2006) 603.

[12] A. Calderón et al., "Amorphous Silicon Position Detectors for the Link Alignment System of the CMS Detector: Users Handbook", Informe Técnico Ciemat 1126, December 2007.

[13] C. Kholer et al., Nucl. Instr. and Methods A 608 (2009) 56.

[14] Sakae Tsushin Kogyo Co., Ltd. - Trade Dept. 322 Ichinotsubo, Nakahara-ku, Kawasaki-city, Kanagawa-prefecture, 211-0016 Japan.

(htpp://www.sakae-tsushin.co.jp).

[15] Omron Corporation, Tokyo Head Office, 3-4-10 Toranomon Minato-ku, Tokyo 105, Japan. (http://www.omron.com).

[16] Applied Geomechanics Incorporated. 1336 Brommer Street, Santa Cruz, CA 95062 USA. (http://www.geomechanics.com/).

[17] J. Alberdi et al., "Tiltmeters for the Alignment System of the CMS Experiment: Users Handbook", Informe Técnico Ciemat 1107, May 2007. 
Table 1: Example of the determination of Ts using the variable $\Delta \mathrm{Z}$ (LD-AR), at $\Phi=$ $315^{\circ}$, in the CMS $+Z$ side, from the CRAFT09 run recorded data. The columns display the following quantities: $\mathrm{t}_{\mathrm{o}}{ }^{\prime}$, mean value, RMS, $\Delta$ min and $\Delta$ max of the corresponding distributions.

\begin{tabular}{|c|c|c|c|c|}
\hline $\mathrm{t}_{\mathrm{o}}{ }^{\prime}(\mathrm{h})$ & Mean $(\mathrm{mm})$ & $\mathrm{RMS}(\mathrm{mm})$ & $\Delta \min (\mathrm{mm})$ & $\Delta \max (\mathrm{mm})$ \\
\hline 0 & -0.054 & 0.015 & -0.068 & 0.001 \\
\hline 2 & -0.029 & 0.011 & -0.040 & 0.001 \\
\hline $\mathbf{3}$ & -0.023 & 0.009 & $\mathbf{- 0 . 0 3 3}$ & 0.001 \\
\hline 4 & -0.022 & 0.008 & -0.030 & 0.000 \\
\hline
\end{tabular}

Table 2: Results from the stabilization calculations in both CRAFT08 and CRAFT09 runs: the variable under observation is displayed in the first column; the Ts time for that variable in the CRAFT08 run is given in the second column while in the third column the result for the CRAFT09 run is shown.

\begin{tabular}{|c|c|c|}
\hline Variable & CRAFT08 Ts (h) & CRAFT09 Ts (h) \\
\hline$\Delta \mathrm{Z}(\mathrm{LD}-\mathrm{AR})$ & $3.5 \pm 0.5$ & $2.5 \pm 0.5$ \\
\hline$\Delta \mathrm{Z}(\mathrm{TP}-\mathrm{ME} 1 / 1)$ & $10.5 \pm 0.5$ & $0.5 \pm 0.5$ \\
\hline$\Delta \mathrm{R}(\mathrm{MAB}-\mathrm{ME} 1 / 2)$ & $2.5 \pm 0.5$ & $1.5 \pm 0.5$ \\
\hline$\Delta \mathrm{R}(\mathrm{TP}-\mathrm{ME} 1 / 2)$ & $0.5 \pm 0.5$ & $0.5 \pm 0.5$ \\
\hline$\Delta \Phi(\mathrm{MAB})$ & $\mathbf{1 3 . 5} \pm \mathbf{0 . 5}$ & $12.5 \pm 0.5$ \\
\hline
\end{tabular}


Table 3: Maximum expected motions in $\Delta \mathrm{Z}, \Delta \mathrm{R}$ and $\Delta \Phi$ after $\mathrm{Ts}=13.5 \mathrm{~h}$ calculated from the Link Alignment System data recorded in the CRAFT08 and CRAFT09 runs.

\begin{tabular}{|c|c|c|}
\hline Variable & CRAFT08 & CRAFT09 \\
\hline$\Delta \mathrm{Z}(\mathrm{LD}-\mathrm{AR})$ & $\pm 14 \mu \mathrm{m}$ & $\pm 12 \mu \mathrm{m}$ \\
\hline$\Delta \mathrm{Z}(\mathrm{TP}-\mathrm{ME} 1 / 1)$ & $\pm 26 \mu \mathrm{m}$ & $\pm 8 \mathrm{~m}$ \\
\hline$\Delta \mathrm{R}(\mathrm{MAB}-\mathrm{ME} 1 / 2)$ & $\pm 10 \mu \mathrm{m}$ & $\pm 23 \mu \mathrm{m}$ \\
\hline$\Delta \mathrm{R}(\mathrm{TP}-\mathrm{ME} 1 / 2)$ & $\pm 31 \mu \mathrm{m}$ & $\pm 26 \mu \mathrm{rad}$ \\
\hline$\Delta \Phi(\mathrm{MAB})$ & $\pm 30 \mu \mathrm{rad}$ & \\
\hline
\end{tabular}

Table 4: Link Alignment System monitored periods in 2010, during the pp $7 \mathrm{TeV}$ collisions data taking. One or several magnet on/off operations were done in between periods. First data in each of the monitored periods was recorded $24 \mathrm{~h}$ after the $\mathrm{B}=3.8$ $\mathrm{T}$ field strength was reached.

\begin{tabular}{|c|c|c|c|}
\hline Label for period & $\begin{array}{c}\text { Period last } \\
\text { (day/month) }\end{array}$ & $\begin{array}{c}\text { Total number } \\
\text { of days }\end{array}$ & $\begin{array}{c}\text { Recorded } \\
\text { Events }\end{array}$ \\
\hline P1 & $8 / 3-13 / 4$ & 37 & 45 \\
\hline P2 & $16 / 4-22 / 4$ & 7 & 8 \\
\hline P3 & $3 / 5-19 / 5$ & 17 & 44 \\
\hline P4 & $3 / 6-18 / 6$ & 16 & 25 \\
\hline P5 & $9 / 8-14 / 8$ & 6 & 12 \\
\hline P6 & $17 / 8-27 / 8$ & 11 & 18 \\
\hline P7 & $3 / 9-17 / 10$ & 45 & 82 \\
\hline
\end{tabular}


Table 5: Comparison of observations done in the seven monitored time-periods. The spatial positions of the sensors used for the table were:

(1) $+\mathrm{Z}, \Phi=315^{\circ}$; (2) $+\mathrm{Z}, \Phi=135^{\circ}$; (3) $-\mathrm{Z}, \Phi=255^{\circ}$; (4) $-\mathrm{Z}, \Phi=195^{\circ}$; (5) $+\mathrm{Z}$; (6) $+Z$; (7) $+Z$; (8) +Z; (9) $-Z, \Phi=15^{\circ}$.

\begin{tabular}{|cc|c|c|c|c|c|c|c|}
\hline \multicolumn{2}{|c|}{$\begin{array}{c}\text { Period } \\
\text { Observable }\end{array}$} & $\mathrm{P} 1$ & $\mathrm{P} 2$ & $\mathrm{P} 3$ & $\mathrm{P} 4$ & $\mathrm{P} 5$ & $\mathrm{P} 6$ & $\mathrm{P} 7$ \\
\hline$(1)$ & $\Delta \mathrm{Z}(\mathrm{LD}-\mathrm{AR})(\mu \mathrm{m})$ & $-137 \pm 62$ & $-40 \pm 36$ & $32 \pm 41$ & $-51 \pm 29$ & $-8 \pm 6$ & $-37 \pm 15$ & $-42 \pm 35$ \\
\hline$(2) \Delta \mathrm{Z}(\mathrm{TP}-\mathrm{ME} 11)(\mu \mathrm{m})$ & $11 \pm 6$ & $-2 \pm 7$ & $10 \pm 7$ & $14 \pm 10$ & $1 \pm 1$ & $-10 \pm 4$ & $12 \pm 7$ \\
\hline$(3) \Delta \mathrm{R}(\mathrm{TP}-\mathrm{ME} 12)(\mu \mathrm{m})$ & $10 \pm 11$ & $-5 \pm 8$ & $-13 \pm 13$ & $-10 \pm 10$ & $-1 \pm 6$ & $-3 \pm 7$ & $7 \pm 12$ \\
\hline$(4)$ & $\Delta \mathrm{R}(\mathrm{LD}-\mathrm{TP})(\mu \mathrm{m})$ & $-27 \pm 14$ & $-7 \pm 4$ & $-8 \pm 6$ & $-12 \pm 6$ & $-2 \pm 1$ & $-8 \pm 4$ & $-21 \pm 11$ \\
\hline$(5)$ & $\Delta \Phi(\mathrm{AR})(\mu \mathrm{rad})$ & $-11 \pm 10$ & $3 \pm 15$ & $15 \pm 17$ & $-4 \pm 13$ & $1 \pm 2$ & $-3 \pm 2$ & $5 \pm 7$ \\
\hline$(6)$ & $\Delta \Theta(\mathrm{AR})(\mu \mathrm{rad})$ & $-24 \pm 15$ & $-6 \pm 9$ & $-10 \pm 8$ & $-5 \pm 4$ & $1 \pm 2$ & $3 \pm 2$ & $-14 \pm 5$ \\
\hline$(7)$ & $\Delta \Phi(\mathrm{LD})(\mu \mathrm{rad})$ & $-5 \pm 6$ & $0 \pm 5$ & $7 \pm 5$ & $5 \pm 5$ & $-0 \pm 1$ & $-1 \pm 1$ & $8 \pm 8$ \\
\hline$(8)$ & $\Delta \Theta(\mathrm{LD})(\mu \mathrm{rad})$ & $-5 \pm 8$ & $3 \pm 4$ & $-2 \pm 3$ & $-11 \pm 6$ & $1 \pm 2$ & $-0 \pm 2$ & $-14 \pm 6$ \\
\hline$(9)$ & $\Delta \Phi(\mathrm{MAB})(\mu \mathrm{rad})$ & $2 \pm 2$ & $1 \pm 1$ & $0 \pm 1$ & $2 \pm 1$ & $-0 \pm 1$ & $1 \pm 1$ & $3 \pm 1$ \\
\hline
\end{tabular}




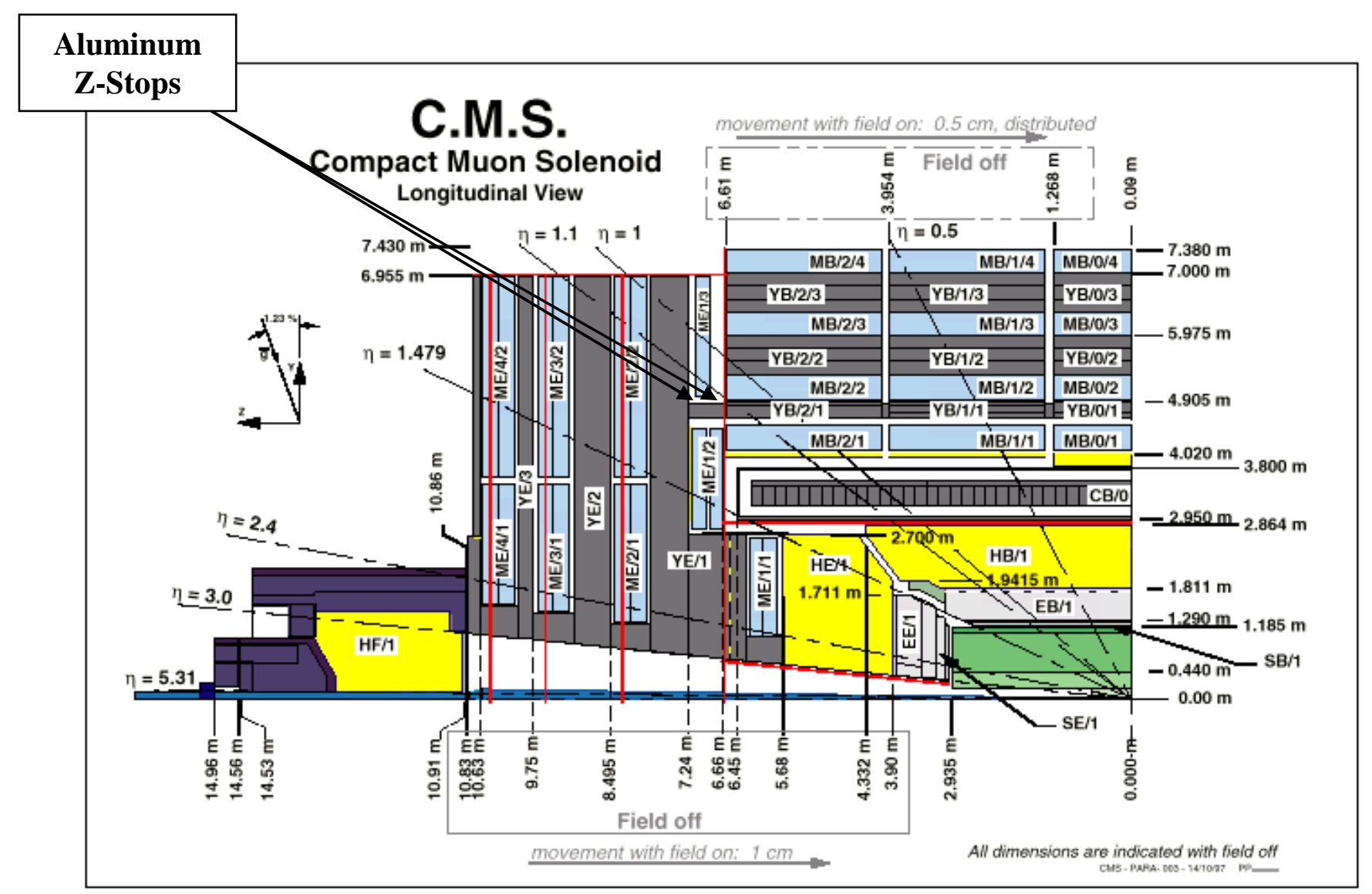

Fig. 1: Longitudinal view (for $+Z$ side and $\Phi=90^{\circ}$ ) of one quadrant of the CMS detector. The approximate positions of the Z-stops in that view are depicted with arrows. 

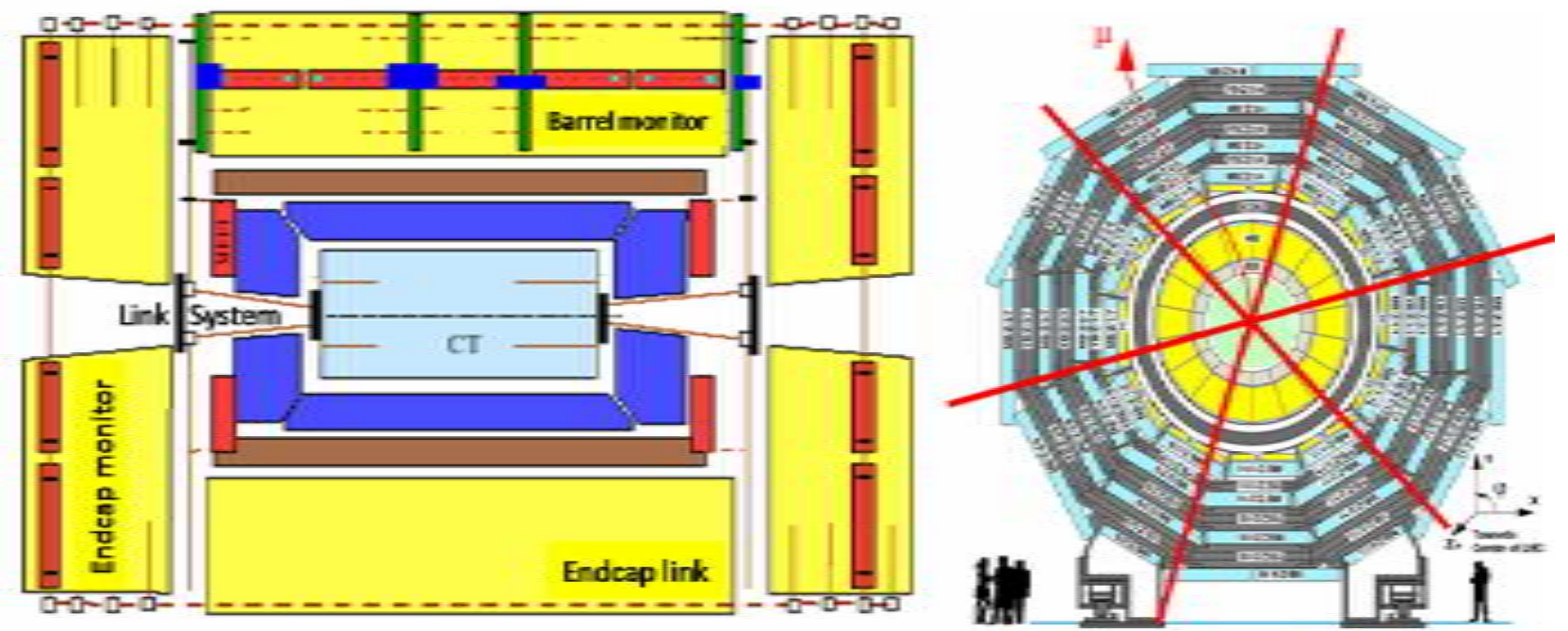

Fig. 2: Schematic view of the Alignment System. Left: one $\Phi$ alignment plane. The continuous and dotted lines show different optical paths. Right: transverse view of the barrel muon detectors. The crossing lines indicate the three $\mathrm{R}-\mathrm{Z}$ alignment $\Phi$ planes. The CMS coordinates-system is also indicated on this side of the figure. 


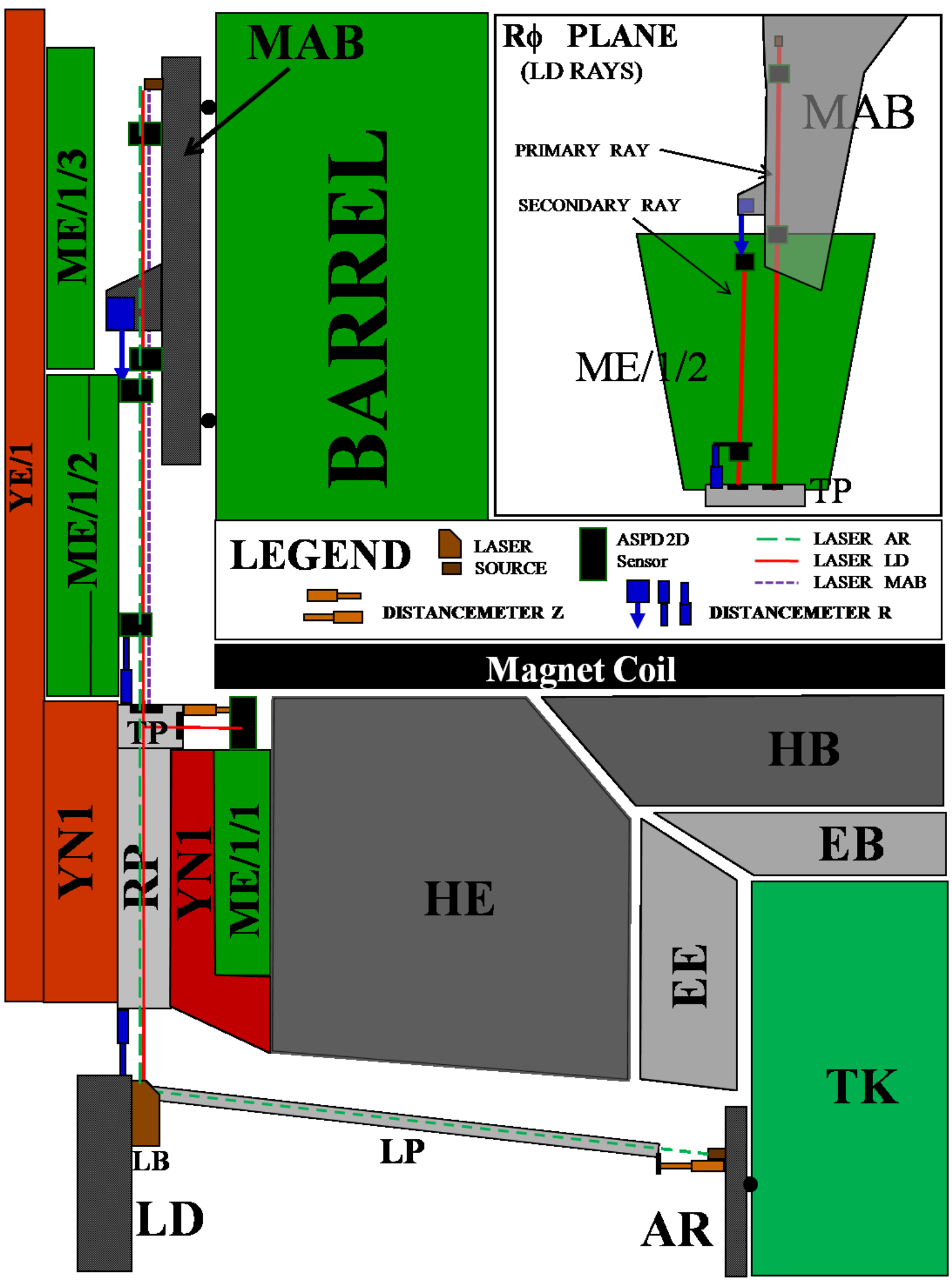

Fig. 3: Link Alignment elements in a quadrant of $\Phi$ plane. 


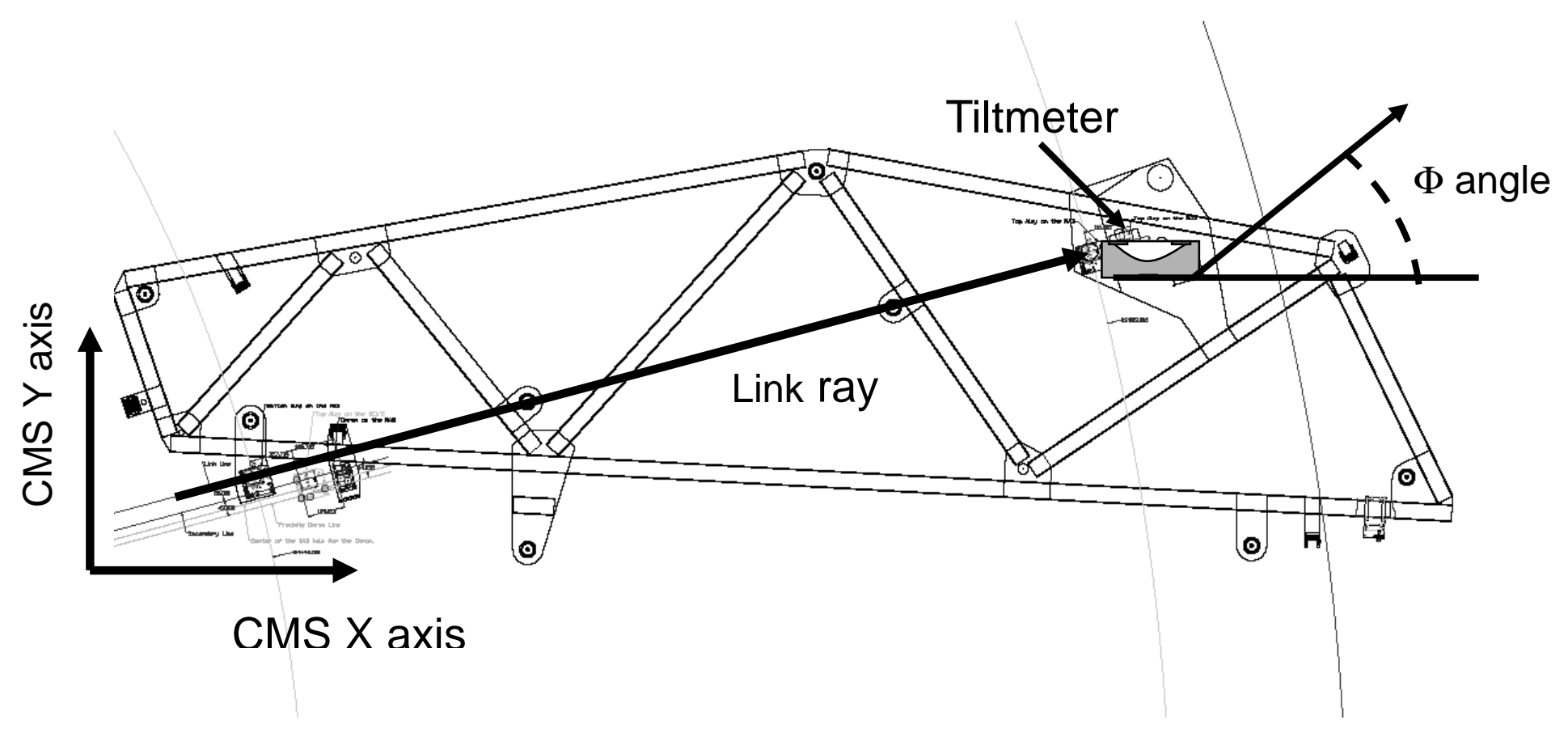

Fig. 4: Sketch of a MAB structure showing the position of the 1D tiltmeter for $\Phi$ monitoring. 



Fig. 5: Link Alignment data from CRAFT08 and CRAFT09 runs, respectively:

a), b): magnetic field intensity as a function of the event number

c), d): $\Delta \mathrm{Z}(\mathrm{LD}-\mathrm{AR})$ relative displacement with respect to the first recorded data, as a function of the data number, for $\Phi=75^{\circ}$, at the CMS $+Z$ side.

e), f): $\Delta Z(T P$ - ME1/1) relative displacement with respect to the first recorded data, as a function of the data number, for $\Phi=15^{\circ}$, at the CMS $-\mathrm{Z}$ side. 

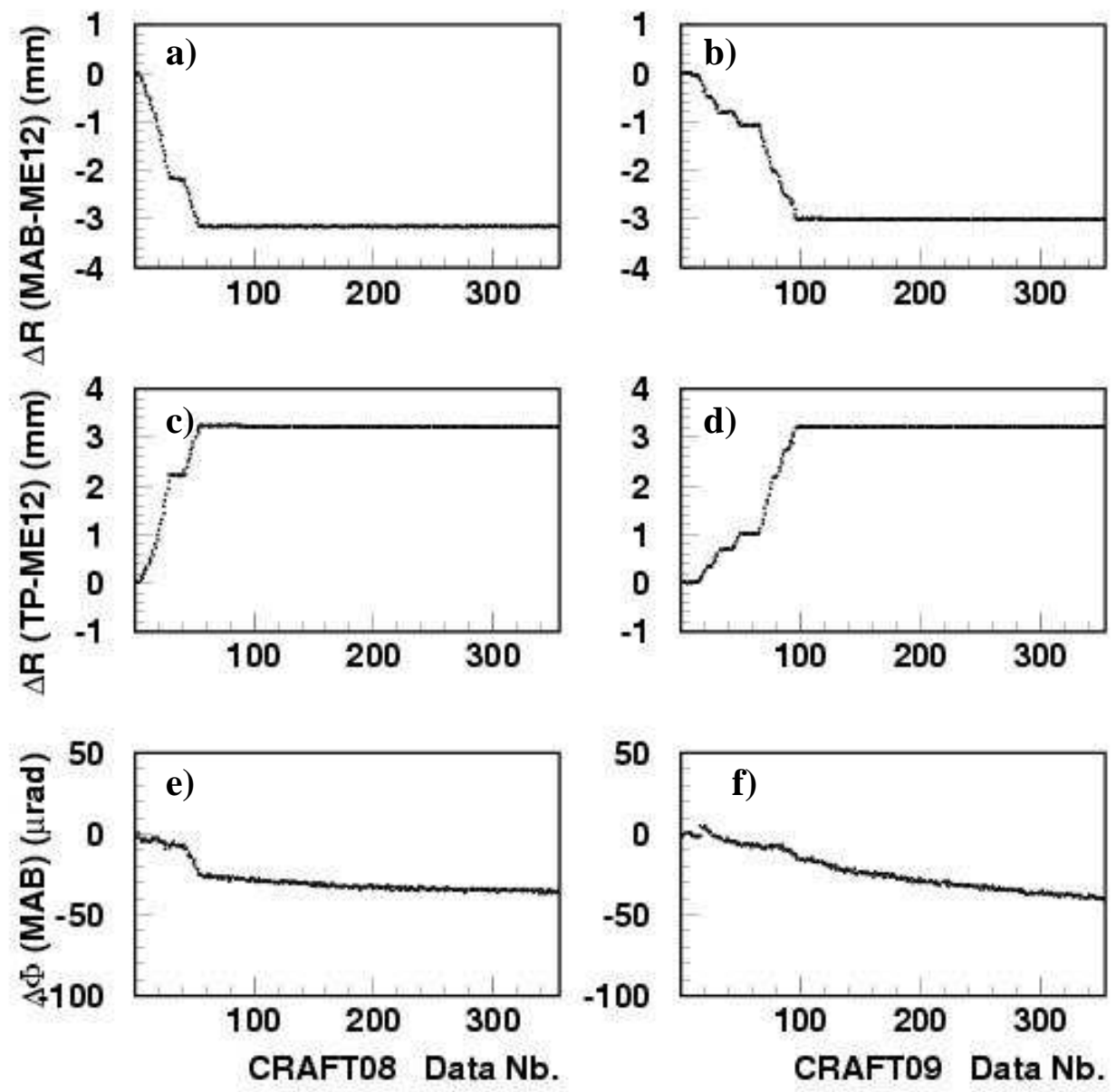

Fig. 6: Link Alignment data from CRAFT08 and CRAFT09 runs, respectively:

a), b): $\triangle \mathrm{R}(\mathrm{MAB}-\mathrm{ME1/2})$ relative displacement with respect to the first recorded data, as a function of the data number, for $\Phi=15^{\circ}$, at the CMS $+Z$ side. c), d): $\Delta \mathrm{R}(\mathrm{TP}-\mathrm{ME} 1 / 2)$ relative displacement with respect to the first recorded data, as a function of the data number, for $\Phi=15^{\circ}$, at the CMS $-\mathrm{Z}$ side.

e), f): $\Delta \Phi(\mathrm{MAB})$ relative tilt with respect to the first recorded data, as a function of the data number, for $\Phi=15^{\circ}$, at the CMS $+Z$ side. 

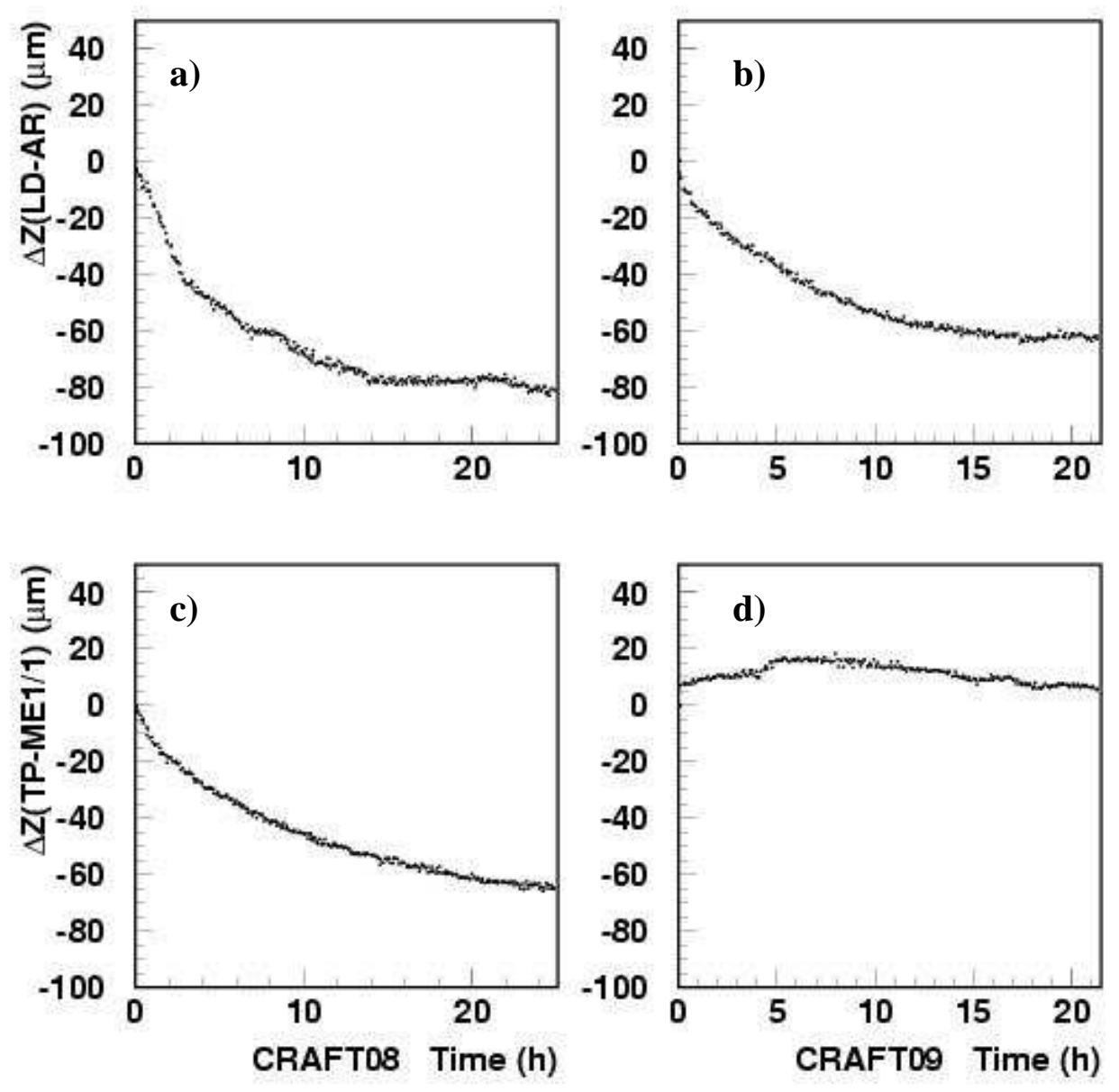

Fig. 7: Link Alignment data from CRAFT08 and CRAFT09 runs, respectively:

a), b): $\triangle \mathrm{Z}(\mathrm{LD}-\mathrm{AR})$ relative displacement with respect to the first recorded data at $\mathrm{B}=3.8 \mathrm{~T}$, as a function of time, for $\Phi=75^{\circ}$, at the $\mathrm{CMS}+\mathrm{Z}$ side.

c), $\mathrm{d}): \Delta \mathrm{Z}(\mathrm{TP}-\mathrm{ME1} / 1)$ relative displacement with respect to the first recorded data at $\mathrm{B}=3.8 \mathrm{~T}$, as a function of tine, for $\Phi=315^{\circ}$, at the $\mathrm{CMS}-\mathrm{Z}$ side. 

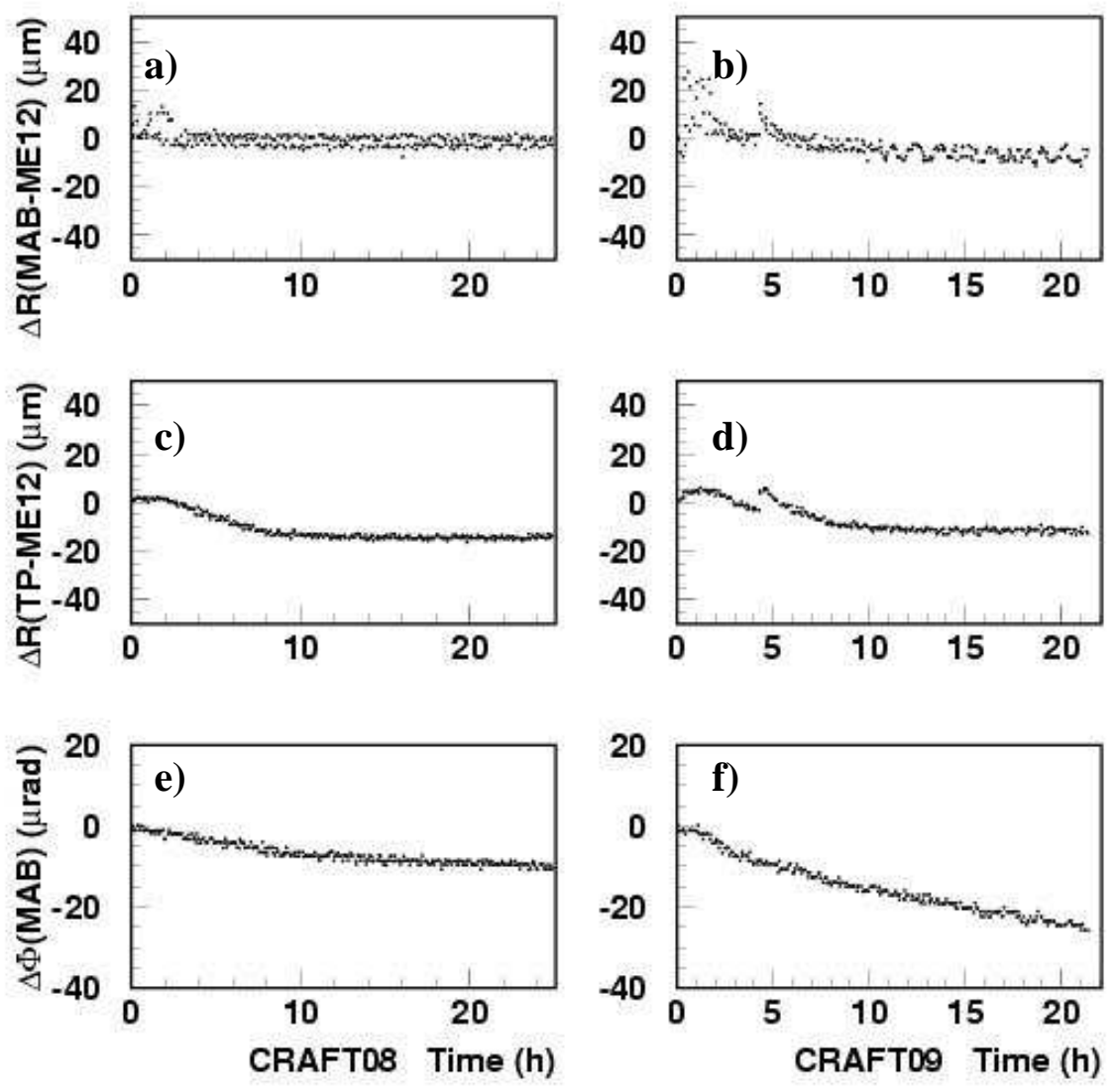

Fig. 8: Link Alignment data from CRAFT08 and CRAFT09 runs, respectively:

a), b): $\triangle \mathrm{R}(\mathrm{MAB}-\mathrm{ME1/2})$ relative displacement with respect to the first recorded data at $\mathrm{B}=3.8 \mathrm{~T}$, as a function of time, for $\Phi=15^{\circ}$, at the $\mathrm{CMS}+\mathrm{Z}$ side.

c), d): $\Delta \mathrm{R}(\mathrm{TP}-\mathrm{ME} 1 / 2)$ relative displacement with respect to the first recorded data at $\mathrm{B}=3.8 \mathrm{~T}$, as a function of time, for $\Phi=15^{\circ}$, at the CMS $-\mathrm{Z}$ side.

e), $\mathrm{f}): \Delta \Phi(\mathrm{MAB})$ relative tilt with respect to the first recorded data at $\mathrm{B}=3.8 \mathrm{~T}$, as a function of time, for $\Phi=15^{\circ}$, at the CMS $+\mathrm{Z}$ side. 

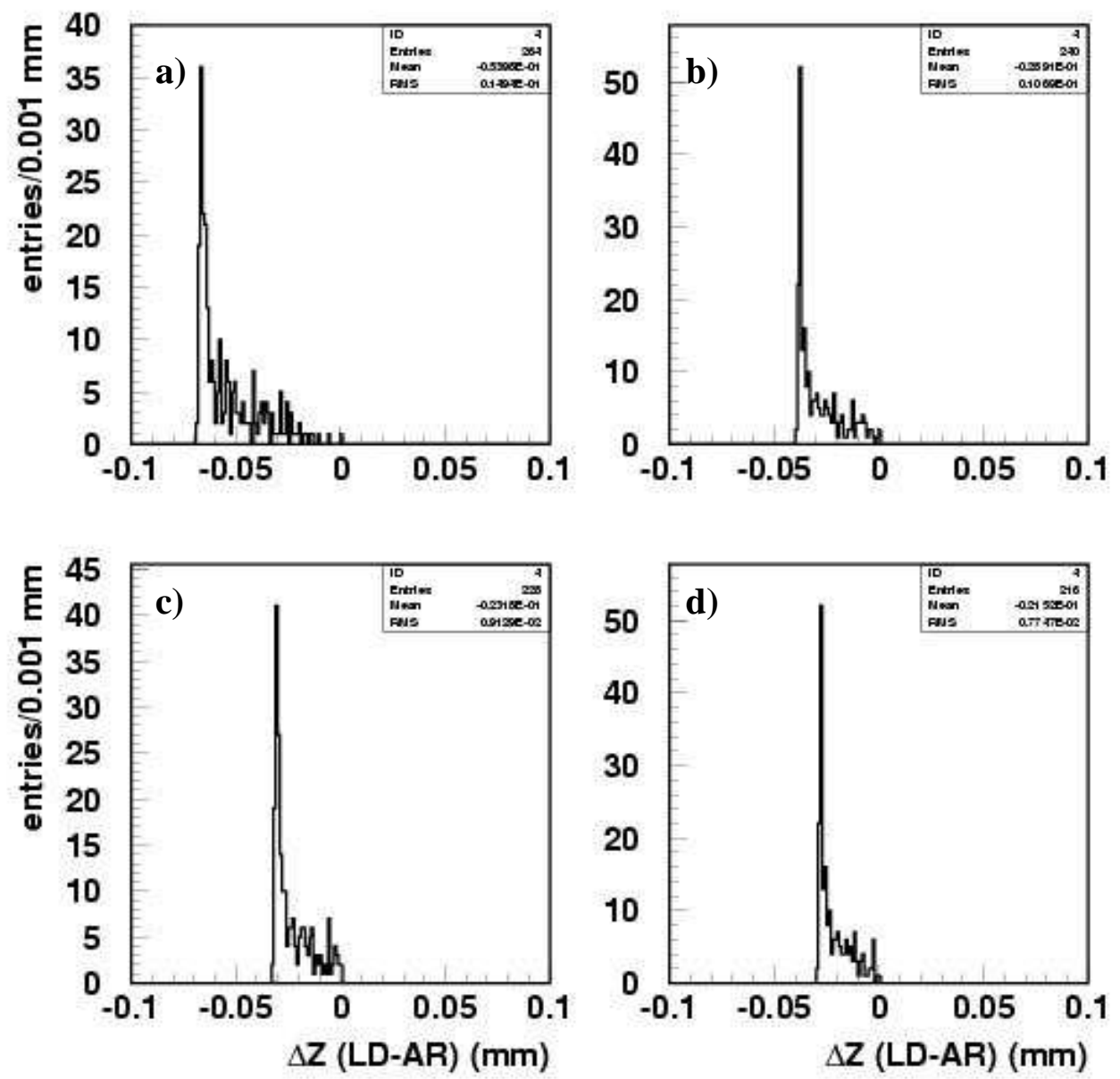

Fig. 9: Link Alignment data from CRAFT09 run: $\Delta \mathrm{Z}(\mathrm{LD}-\mathrm{AR})$ distributions at $\Phi=$ $315^{\circ}$, +Z CMS side, for:
a) $t_{0}^{\prime}=0$ hours,
b) $t_{0}{ }^{\prime}=2$ hours,
c) $t_{0}{ }^{\prime}=3$ hours,
d) $t_{o}{ }^{\prime}=4$ hours. 

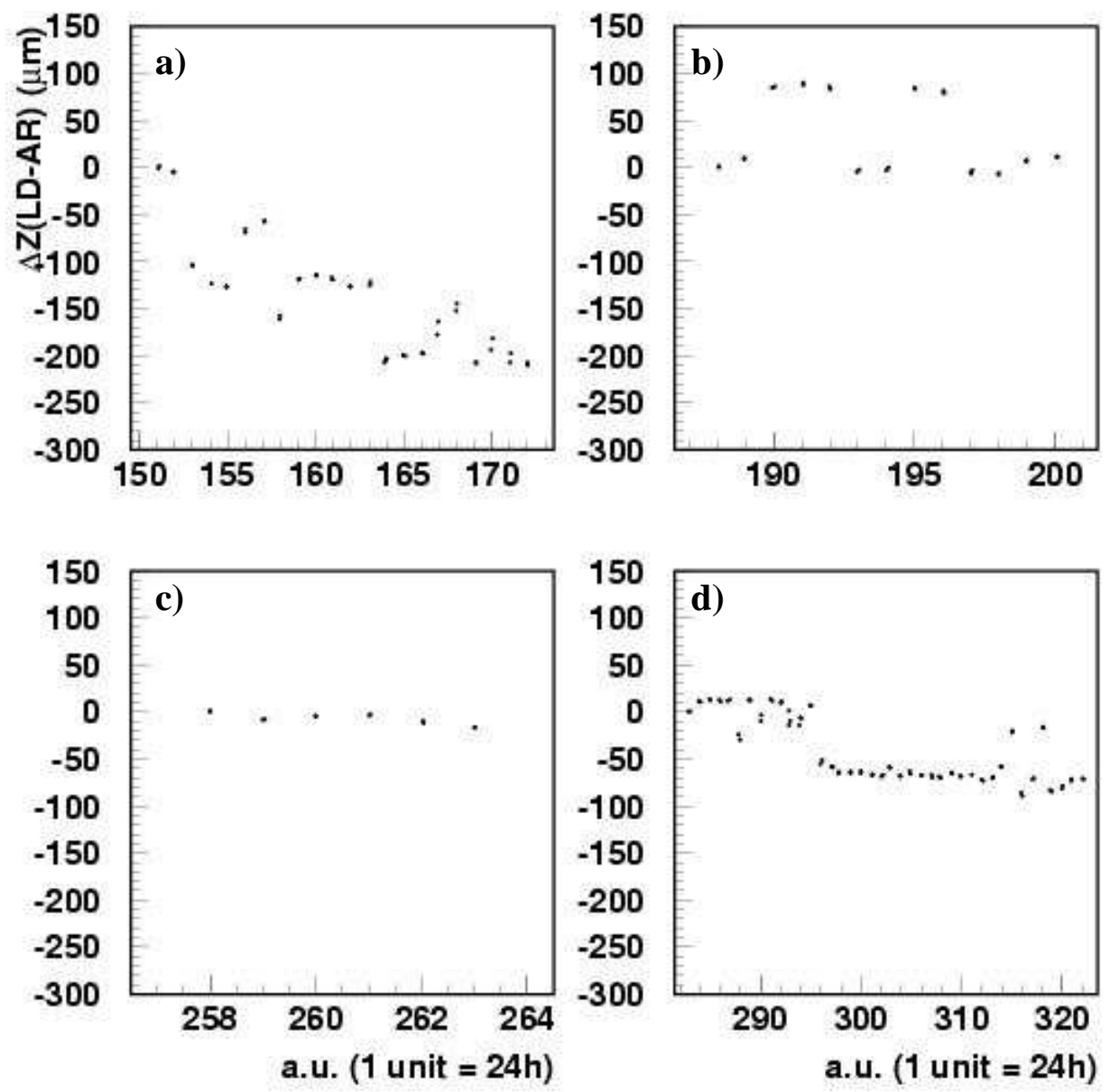

Fig. 10: Measurements of $\Delta \mathrm{Z}(\mathrm{LD}-\mathrm{AR})$ distributions at $\Phi=315^{\circ}, \mathrm{Z} \mathrm{CMS}$ side (always with respect to the sensor value at event number one in each period) for the periods a) P1, b) P3, c) P5 and d) P7, as a function of a proper to the Link System internal run number (a.u. in the figure). Each a.u. has a time-length of 1 day. A maximum of two events are recorded each day. In a few Link runs no data taking took place. Most of the black dots are the superposition of two events recorded in a given Link run. 

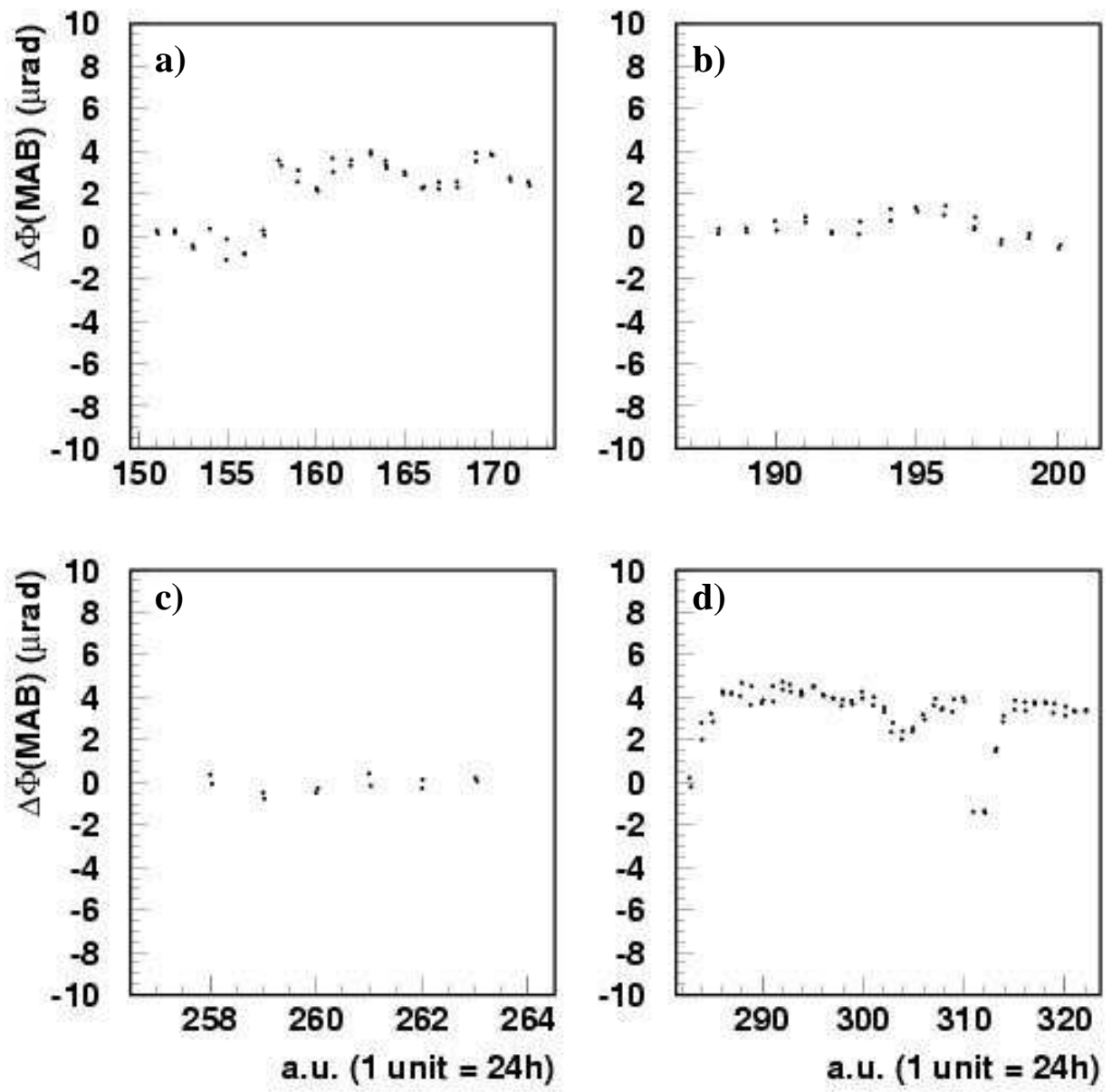

Fig. 11: Measurements of $\triangle \Phi(\mathrm{MAB})$ distributions from the tiltmeter on the $\mathrm{MAB}$ at $\left(-Z, \Phi=15^{\circ}\right)$, always with respect to the sensor value at event number one in each data sample, for the periods a) P1, b) P3, c) P5 and d) P7, as a function of a proper to the Link System internal run number (a.u. in the figure). Each a.u. has a time-length of 1 day. A maximum of two events are recorded each day. In a few Link runs no data taking took place. Most of the black dots are the superposition of the two events recorded in a given Link run. 